\title{
Rising risks of late-spring frosts in a changing climate
}

To the Editor — Late-spring frosts (LSFs) - frost events occurring after germination and budburst of herbaceous and woody plants, respectively - have an important ecological and economic impact on agriculture and forestry in temperate and boreal regions of the world. The damage induced by LSFs to vulnerable plant organs markedly affects growth, health, competitive ability and distribution limits of plants, and, in North America and Europe, causes more economic losses to agriculture than any other climate-related hazards ${ }^{1,2}$. While attribution of a single LSF episode to climate change is difficult, both the frequency and the severity of damage due to LSF may be amplified by the climate crisis. Furthermore, attempts to mitigate LSF damage and climate change impacts at both shorter and longer timescales may only exasperate the issues.

From early April 2021, frost and freezing temperatures caused severe damage to crops including vineyards and fruit trees, oilseed rapes, potatoes and sugar beets across Europe. In France, about $80 \%$ of the country's wine and fruit tree regions were affected (Box 1), with the extent of the damage also substantial in Italy, the Czech Republic and the UK. The burden of this disaster, which threatens livelihoods and food security, is worsened for producers already suffering due to COVID-19.

Climate change can lead to altered patterns and increased incidences of extreme events ${ }^{3}$, and has already triggered advances in plant spring phenology $y^{4-6}$ (for example, spring leaf unfolding advanced by almost two weeks from 1982-2011 in western Europe ${ }^{7}$ ), with this trend projected to continue throughout the twenty-first century. Research demonstrates that LSF damage, resulting from frost events overlapping with accelerated phenology, has increased on temperate and boreal vegetation over middle latitudes across $43 \%$ in the Northern Hemisphere, especially in Europe between 1982-2012 (ref. ${ }^{8}$ ), and has also increased on woody plants in the state of Illinois (USA) during the last 124 years $^{4}$. Future projections show that over one-third, one-fourth and one-tenth of the European, Asian and North American temperate forest areas, respectively, will be subjected to increasing damage of LSFs ${ }^{9}$.

\section{Box 1 | The dilemma of short-term adaptation measures to limit the damage of LSFs to crops}

Due to fears of crop losses and the consequent socio-economic impact, many wine and fruit producers in France and Europe have adopted reactive strategies to combat LSFs in their vineyards and fruit orchards. These strategies consisted in the use of sprinklers, surface irrigation, wind machines, helicopters, solid and liquid fuel heating, including candle lighting, paraffin wax, wood, hay bale and crop residue burning in vineyards and orchards (see figure within this box).

While irrigation, wind machines and helicopters are a common means of mitigating damage due to LSFs, they are costly and energy intensive. In addition, using water to alleviate frost damage can reduce water availability for later use, which may be of concern in the case of adjudicated surface water and regulated groundwater resources. Wood, hay bale and crop residue burnings in vineyards and orchards cause numerous environmental problems, such as the emission of greenhouse gases that contribute to global warming, increased levels of particulate matter and smog that cause health hazards, loss of biodiversity of agricultural lands and the deterioration of soil fertility.

The French government has declared an 'agricultural disaster' and pledged $€ 1$ billion in aid measures to help wine and fruit growers.

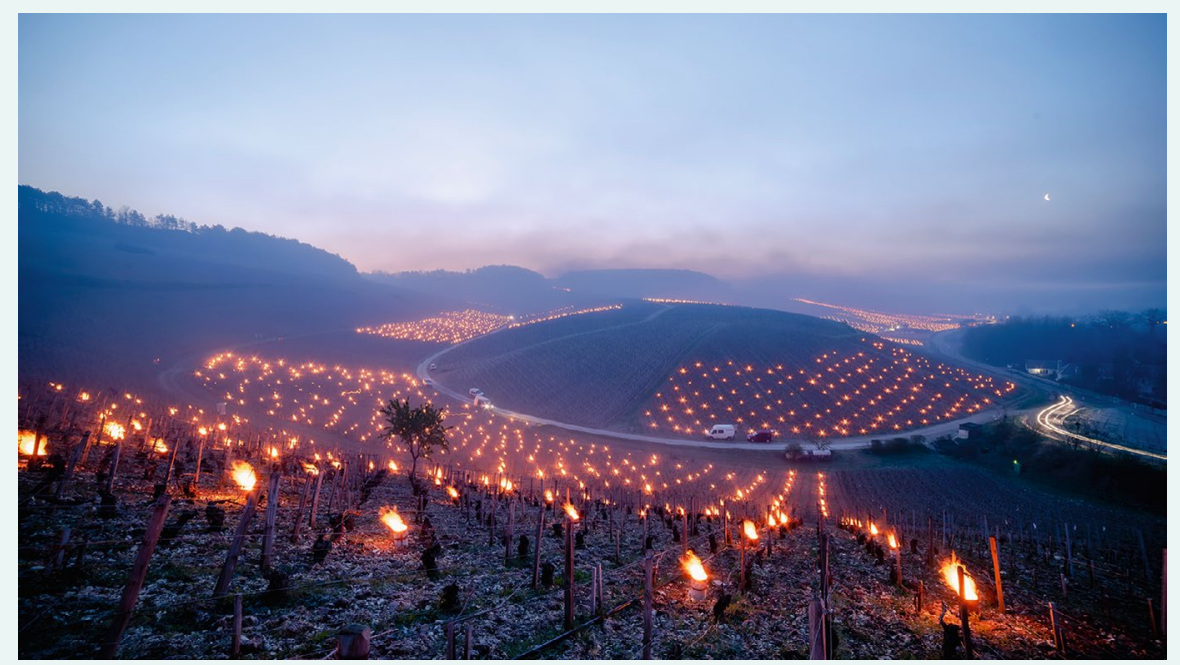

An aerial view of smoke derived from fires lit in the vineyards to protect them from frost at the heart of Chablis, the Bourgogne-Franche-Comté region, on 6 April 2021. Photo courtesy of Titouan Rimbault.

Because LSF damage occurs as a result of a complex interaction between biological factors, frost events and other abiotic influences, frost risk can be highly variable and difficult to predict. For example, LSF damage remained mostly unchanged in lower altitudes in Switzerland (over the $1975-2016$ period) ${ }^{10}$ but increased markedly at stations located at elevations higher than $800 \mathrm{~m}$ above sea level, with differences attributed to both shifts in frost dates and stronger phenological shifts at higher elevations ${ }^{10}$. Climate change itself is also complex: milder winter temperatures can influence the depth and duration of snow cover that protect roots from frosts through insulation, thus limiting damage ${ }^{11}$.

LSF events can be economically costly a single LSF event across Europe in spring 2017 resulted in economic losses of $€ 3.3$ billion, with only $18 \%$ of the crops insured ${ }^{12}$ 
- but can also have further implications for food security, plant productivity and ecological interactions. For example, the flowering and budburst of perennial herbaceous wildflower species can be affected by LSFs ${ }^{13}$, in turn impacting on pollinators and herbivores. LSF damage in the USA over the 1979-2010 period resulted in lower vegetation growth in spring and subsequent lower vegetation greenness in summer ${ }^{14}$.

Methods to limit LSF damage in the short-, medium- and long-term are necessary, but have the potential to further worsen environmental issues instead of providing sustainable adaptations. For example, in response to the April 2021 frost events, farmers used irrigation, solid and liquid fuel heating and burning in vineyards and orchards (Box 1).

Medium-term strategies include the selection of more favourable growing regions, shifting sowing dates and crop rotations (for annual crops), and changing to resistant species or cultivars. Similarly, long-term adaptation strategies include breeding for varieties with late budburst (for woody crops) and those better adapted to thermal shocks, including LSFs. While current breeding strategies for climate-resilient crops are more focused toward selecting crops for heat or drought rather than frost tolerance, breeding for improved frost tolerance would not only reduce direct frost damage but also allow crops to be sown earlier, thereby alleviating risks of late-season drought and/or heat stresses $^{15}$. Early sowings represent an important adaptation strategy to climate change for annual crops across temperate regions to escape summer drought and heat stresses, which represent the most important yield-limiting factors for key arable crops ${ }^{6,16,17}$.

The ongoing damage due to LSFs represents an unprecedented disaster for the agricultural sector, with substantial ecological and economic impacts for society at large. At the same time, such catastrophic events represent an opportunity for transformative changes in how science and policy should come together to address some of the key issues of twenty-first century agriculture, namely maintaining or raising agricultural productivity, reducing the negative environmental impact of agriculture and adapting agriculture to ongoing climate change. This can be done in three ways: (i) awareness raising to foster best management practices affecting short-term LSF management strategies; (ii) region-specific risk assessment of the frequency and severity of LSFs to inform decision-making in land management, forestry, agriculture and insurance policy; and (iii) breeding new crop varieties that help diversify current cropping systems based on specific soil and climatic conditions of a given region.
Jay Ram Lamichhane (D) $\square$

AGIR, University of Toulouse, INRAE,

Castanet-Tolosan, France.

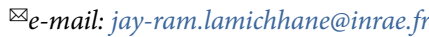

Published online: 1 July 2021

https://doi.org/10.1038/s41558-021-01090-X

References

1. Snyder, R. L. \& de Melo-Abreu, J. P. Frost Protection: Fundamentals, Practice, and Economics (FAO, 2005).

2. Papagiannaki, K. et al. Nat. Hazards Earth Syst. Sci. 14, 2375-2386 (2014)

3. Herring, S. C. et al. Bull. Am. Meteorol. Soc. 96, S1-S172 (2015).

4. Augspurger, C. K. Ecology 94, 41-50 (2013).

5. Unterberger, C. et al. PLoS ONE 13, e0200201 (2018).

6. Pulatov, B. et al. Agric. For. Meteorol. 214, 281-292 (2015).

7. Fu, Y. H. et al. Glob. Ecol. Biogeogr. 23, 1255-1263 (2014).

8. Liu, Q. et al. Nat. Commun. 9, 426 (2018).

9. Zohner, C. M. et al. Proc. Natl Acad. Sci. USA 117, 12192-12200 (2020).

10. Vitasse, Y. et al. Agric. For. Meteorol. 248, 60-69 (2018).

11. Tierney, G. L. et al. Biogeochemistry 56, 175-190 (2001).

12. Faust, E. \& Herbold, J. Spring frost losses and climate change not a contradiction in terms. Munich Re https://bit.ly/3x3UQXS (2018).

13. Inouye, D. W. Ecology 89, 353-362 (2008).

14. Kim, Y. et al. Agric. For. Meteorol. 194, 132-143 (2014).

15. Zheng, B. et al. J. Exp. Bot. 66, 3611-3623 (2015).

16. Fahad, S. et al. Front. Plant Sci. 8, 1147 (2017).

17. Donatelli, M. et al. Environ. Res. Lett. 10, 75005 (2015).

\section{Acknowledgements}

J.R.L. is supported by UMT PACTOLE, a multi-partnership initiative that aims to produce knowledge and tools useful for the diversification of cropping systems based on oilseeds and pulses, with a twofold objective: an improved adaptation to climate change as well as agro-ecological and nutritional transition.

Competing interests

The authors declare no competing interests. 